\title{
A fatal case of dissecting aortic root aneurysm due to undiagnosed Marfan syndrome in the puerperium
}

\author{
A A N Nishad ${ }^{1}$, R Herath ${ }^{1}$, G R C Silva ${ }^{1}$, R Mohommad ${ }^{1}$, P Wijesinghe ${ }^{1}$, S Padumadasa ${ }^{1}$, A Premawardhena ${ }^{1}$, \\ R Premarathne ${ }^{2}$
}

Sri Lanka Journal of Obstetrics and Gynaecology 2012; 34: 55-57

\begin{abstract}
Aortic dissection in pregnancy is a lethal cardiovascular complication in women with Marfan syndrome. The course of the treatment would be decided upon by the size of aortic root dilation. We report a case where the unawareness of the diagnosis of Marfan syndrome antenatally led to disastrous post partum consequences even after an uneventful childbirth.
\end{abstract}

Key words: Marfan syndrome, dissecting aneurism

\section{Introduction}

Marfan syndrome is one of commoner autosomal dominant single gene malformation syndromes that affect both sexes equally in any ethnic group. It is a multisystem disorder resulting in diminished strength of connective tissues mostly affecting cardiovascular, ocular and skeletal systems. The cardiovascular complications include sudden arrhythmias, mitral valve prolapse with or without regurgitation, left ventricular dilatation and cardiac failure, pulmonary artery dilatation, aortic root dilatation. Dilatation of aortic root leads to aortic valve incompetence and predisposes to dissection of aorta especially when the diameter at the Sinus of Valsalva exceeds $4.5 \mathrm{~cm}^{1}$.

In pregnancy and puerperium the risk of aortic dissection is increased, probably due to inhibition of collagen and elastin deposition in the aorta by oestrogens and the hyperdynamic hypervolaemic circulatory state.

\section{Case report}

A 37-year old woman presented in her fifth pregnancy with four previous uneventful

\footnotetext{
${ }^{1}$ Professorial Obstetrics and Gynaecology Unit,

${ }^{2}$ Professorial Medical Unit, North Colombo Teaching Hospital, Ragama, Sri Lanka.

Correspondence: A A N Nishad

E-mail: aanilnga@gmail.com
}

pregnancies. Her height was $166 \mathrm{~cm}$. There were no obvious cardiovascular or skeletal abnormalities noted. Following an uneventful antenatal period she went into spontaneous labour at term and delivered a healthy baby. She developed a blood and mucus diarrhoea from the day of delivery, which settled after six days. Sigmoidoscopy with biopsy, and stool culture for salmonella were normal.

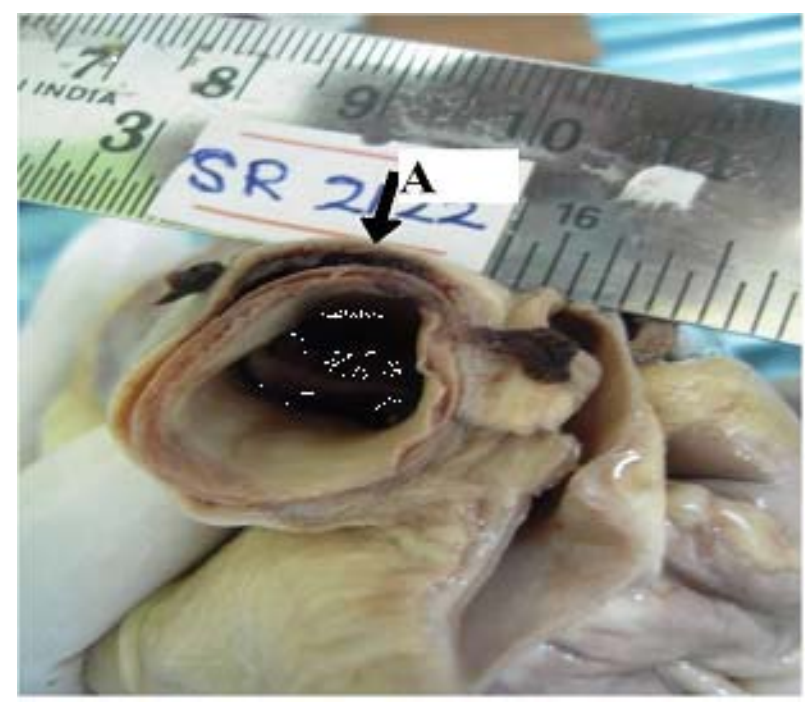

Figure 1. The macroscopic appearance (A) of the aortic dissection.

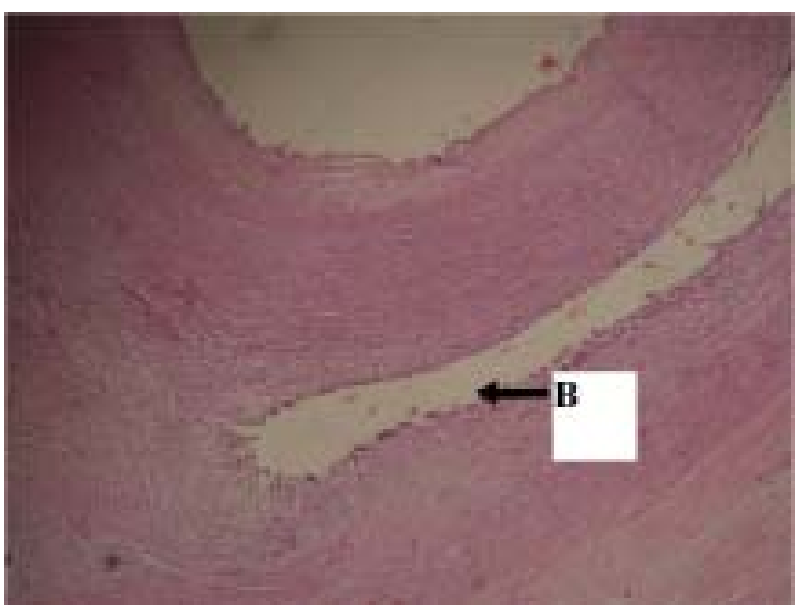

Figure 2. The microscopic appearance (B) of the aortic dissection. 
On the thirteenth postpartum day she was admitted to hospital with worsening shortness of breath. On admission she was found to have tachycardia, low blood pressure, and evidence of pulmonary oedema. Electrocardiogram showed sinus tachycardia and ST depressions change in lateral chest leads (V3-V6) chest leads. She died six hours after admission despite attempted resuscitation and treatment.

Postmortem revealed degeneration of tunica media in the ascending aorta, and a dissecting aneurysm extending up to infundibulum (Figure 1 and 2 ). Furthermore, there were right sided pleural adhesions.

Subsequent inquiry of family members revealed that one of her brothers and one of her children were suffering from Marfan syndrome. Further, closer scrutiny revealed that she had developed a spontaneous pneumothorax at the age of 22 years which was not further investigated.

\section{Discussion}

The positive family history, dissection of the ascending aorta (two major criteria) and history of spontaneous pneumothorax (one minor criterion) fulfilled the Ghent criteria for diagnosis of Marfan Syndrome ${ }^{1}$.

Vigilant attention at the time of the spontaneous pneumothorax and during her pregnancies coupled with family history should have helped to make the diagnosis in this patient at an earlier point of time.

Women with the syndrome should be counselled before conception about the risks of pregnancy to both mother and fetus. Because pre-conceptual dilatation of the ascending aorta is an important predictor for aortic dissection, it should be excluded before pregnancy and if the root of the aorta is more than $45 \mathrm{~mm}$, it is safer to discourage pregnancy.

Once the woman become pregnant her cardiac and aortic root size should be monitored with repeated echocardiography. Prophylactic use of $\beta$-blockers may be useful in preventing further aortic dilatation although Atenolol, in a few studies, showed association with low birth weight for intrauterine exposure ${ }^{1,6}$. Surgical replacement of aortic root should be considered during gestation in patients with progressive aortic dilatation when or before the aortic root reaches $5.5 \mathrm{~cm}$.
Because of the potential risk of ionizing radiation to the fetus, noninvasive methods such as transesophageal echocardiography and magnetic resonance imaging are preferred to contrast aortography for the diagnosis of aortic dissection.

Vaginal delivery can be allowed in patients with the Marfan syndrome who do not have cardiovascular system abnormalities. In patients with aortic root dilatation and aortic dissection, caesarean section can be the preferred method of delivery with control of hypertension and reducing left ventricular contractility with Hydralazine and beta blockers ${ }^{8,1}$.

Another area of concern in this patient is the apparent unmet need for contraception. Appropriate contraceptive advice to space and limit the number of children in this woman certainly would have prevented the unrecognized progression of the condition and the subsequent demise.

\section{Conclusion}

We emphasize the importance of considering illnesses such as Marfan syndrome in females with positive family history even in the absence of telltale features in order to observe appropriate precautions during pregnancy. Educating patients and family members about the nature and inheritance of the disease is important so that the positive family history will be forthcoming.

\section{Acknowledgements}

We would like to acknowledge Dr. W. R. A. S. Rajapaksha (Registrar in Forensic Medicine) for providing the photographs.

\section{References}

1. Elkayam U, Ostrzega E, Shotan A, Mehra A. Cardiovascular problems in pregnant women with Marfan syndrome. Annals of Internal Medicine 1995; 123: 117-22.

2. Dean JCS. Marfan syndrome: clinical diagnosis and management. European Journal of Human Genetics 2007; 15: 724-733.

3. Goland S, Elkayam U. Cardiovascular problems in pregnant women with Marfan syndrome. Circulation 2009; 119: 61923.

4. Lilian JM, Vos FE, Janneke T, Godfried HB, Aeiko HZ, Barbara JM. Pregnancy and aortic root growth in the Marfan syndrome: a prospective study. European Heart Journal 2003; 26: 914-920.

5. Therrien J, Gatzoulis M, Graham T, Bink-Boelkens M, Connelly M, Niwa K, Mulder BJM, Pyeritz RE, Perloff J, Somerville J, Webb GD. Canadian Cardiovascular Society 
Consensus Conference 2001 Update: Recommendations for the Management of Adults with Congenital Heart DiseasePart II. Can J Cardiol 2001; 17: 1029-50.

6. Lardoux H, Gerard J, Blazquez G, Chouty F, Flouvat B. Hypertension in pregnancy: evaluation of two $\beta$-blockers atenolol and labetalol. Eur Heart J 1983; 4(Suppl): 35-40.
7. Gott VL, Greene PS, Alejo DE, et al. Replacement of the aortic root in patients with Marfan's syndrome. N Engl J Med 1999; 340: 1307-13.

8. Eagle KA, DeSanotis R. Diseases of the aorta. In: Heart Disease, Braunwald E, ed. Philadelphia: WB Saunders; 1992: 1528-57. 